\section{Compreensão das prescrições pediátricas de antimicrobianos em Unidades de Saúde em um município do sul do Brasil}

\section{Understanding of pediatric antimicrobial prescriptions at health units in a Southern Brazil city}

\section{Ana Paula Simões Menezes'}

Marlos Rodrigues Domingues"

Ana Luiza Muccillo Baisch"'

'Programa de Pós-Graduação Ciências da Saúde da FURG, Rio Grande, RS e Centro de Ciências da Saúde do Curso de Farmácia da URCAMP, Bagé,RS.

"Programa de Pós-Graduação em Epidemiologia da Universidade Federal de Pelotas, Pelotas, RS.

I"'Departamento de Ciências Fisiológicas, Programa de Pós-Graduação em Ciências da Saúde da FURG, Rio Grande, RS.

\section{Resumo}

O objetivo do estudo foi avaliar a compreensão da prescrição de antimicrobianos por acompanhantes de crianças após consulta pediátrica, descrevendo seu perfil de prescrição em Unidades de Saúde, em um município do sul do Brasil. Trata-se de um delineamento transversal, no qual a população-alvo foram acompanhantes das crianças que tiveram prescrição de antimicrobianos $(n=209)$ entre maio e julho de 2007. Foi gerado um escore de compreensão (4-12 pontos) embasado em afirmativas corretas, incorretas ou ignoradas, relativas às informações da prescrição. A compreensão foi considerada adequada quando o valor do escore foi menor do que 6,5 pontos. Verificou-se que a escolaridade $(p=0,05)$, renda $(p=0,03)$, cor da pele $(p=0,007)$ do acompanhante e o tempo de consulta ( $p$ $=0,05$ ) estiveram diretamente associados com a melhor compreensão da prescrição. A compreensão adequada representou $58,9 \%$ das prescrições. Os antimicrobianos mais prescritos foram a amoxicilina e a associação de sulfametoxazol/trimetoprima. Os resultados do estudo apontam a necessidade da padronização da informação escrita por prescritores e implantação de medidas educativas aos acompanhantes das crianças, tais como o fornecimento de orientações verbais e escritas quanto ao correto uso dos antimicrobianos. Assim, assegura-se uma melhor compreensão da prescrição, o que favorece o sucesso do tratamento, evitando também o fenômeno de resistência microbiana.

Palavras-chave: Compreensão. Prescrição pediátrica. Antimicrobianos. 


\section{Abstract}

The aim of the study was to evaluate the understanding of antimicrobial prescriptions by adults accompanying children, describing the medicine utilization profile at Health Units in a South Brazilian city. A cross-sectional study was carried out with adults accompanying children during appointments that resulted in antimicrobial prescription ( $\mathrm{n}=209$ ), from May to June 2007. A score (4-12) was generated to evaluate the understanding based on correct and incorrect/unknown statements about the prescription. Understanding was considered appropriate when the score was below 6.5 . Schooling ( $\mathrm{p}=0.05)$, income $(\mathrm{p}=0.03)$, skin color ( $\mathrm{p}=0.007$ ) of accompanying person and length of the visit in minutes $(\mathrm{p}=0.05)$ were associated with better understanding of prescriptions. Appropriate understanding represented $58.9 \%$ of prescriptions. The most prescribed antimicrobials were amoxicillin and the Trimethoprim-Sulfamethoxazole association. The results point toward the need for standardization of written information and educational measures such as oral and written orientation to assure a better understanding of prescriptions. In this way, better understanding of prescriptions can be assured, which in turn helps treatment to succeed, and also avoids microbial resistance.

Keywords: Understanding. Pediatric prescription. Antimicrobial.

\section{Introdução}

O uso indiscriminado ou inapropriado de antimicrobianos pode acelerar o desenvolvimento de cepas bacterianas resistentes ${ }^{1}$. Cerca de $75 \%$ destes fármacos são prescritos inapropriadamente e, em média, 50\% dos pacientes tomam estes medicamentos de maneira incorreta ${ }^{2}$. Erros de medicação, expectativas dos prescritores e pacientes e a incompreensão, ou o não cumprimento da terapia medicamentosa, são exemplos de fatores que contribuem para o uso irracional de medicamentos resultando no aumento da resistência microbiana a fármacos - farmacorresistência - e gastos desnecessários no setor público ${ }^{2,3}$.

A não compreensão das informações da prescrição é comum nos serviços de saúde e suas causas podem ser atribuídas a uma linguagem complexa, informações desorganizadas ou implícitas e uma caligrafia inadequada, as quais tornam ilegível a informação escrita, dificultando o seu entendimento ${ }^{3,4}$.

A adesão da criança ao tratamento torna-se complicada porque depende de perspectivas e do relacionamento da família com o médico. A comunicação entre pais e prescritor é elemento fundamental na adesão ao tratamento ${ }^{5}$. Fatores relacionados ao acompanhante da criança, como o baixo nível socioeconômico ${ }^{6}$, e ao prescritor, como fornecimento de prescrição com elevado número de medicamentos ${ }^{7}$, podem dificultar a compreensão da informação escrita e contribuir para o não cumprimento do tratamento terapêutico, colocando em risco a adesão da terapia antimicrobiana infantil.

O presente estudo tem por objetivo avaliar a compreensão da prescrição de antimicrobianos pelo acompanhante da criança, após consulta pediátrica em Unidades de Saúde, em um município da região sul do Brasil e descrever o perfil de prescrição de antimicrobianos.

\section{Métodos}

Foi realizado um estudo transversal no município de Bagé, interior do Estado do Rio Grande do Sul, que possui, segundo o censo de 2007 do Instituto Brasileiro de Geografia e Estatística, uma população de aproximadamente 112 mil habitantes. $O$ estudo abrangeu todos os postos de saúde do nível média complexidade (03), os quais disponibilizam serviço especializado em pediatria, e contam com um total de 10 pediatras. A coleta dos dados compreendeu o período de maio a julho de 2007. A população-alvo foi constituída pelos acompanhantes das 
crianças atendidas pelo pediatra do serviço, as quais receberam prescrição de pelo menos um antimicrobiano.

O cálculo de tamanho de amostra para o estudo de associação levou em conta a variável escolaridade (inferior a 4 anos de idade), com um poder estatístico de $80 \%$ e um nível de confiança de 95\%, um Risco Relativo igual a 2 e uma razão não exposto / exposto de 4/1. Considerando uma prevalência de $16 \%$ entre os não-expostos, o resultado foi de 360 indivíduos. A este número foram adicionados $10 \%$ para perdas e recusas, e $15 \%$ para o controle de fatores de confusão, resultando em uma amostra de 455 indivíduos. Por questões logísticas, optou-se por realizar 50 entrevistas de acompanhantes de crianças após consulta pediátrica por cada pediatra $(\mathrm{n}=10)$ do serviço, totalizando 500 entrevistas.

Foram incluídos no estudo os acompanhantes das crianças com idades entre zero e doze anos e que tiveram prescrição de medicamentos no período do estudo. Os acompanhantes incluídos na amostra foram entrevistados no início do horário de atendimento, diariamente, de acordo com uma sequência de números ímpares do agendamento, até que se completassem 50 entrevistas por pediatra, assegurando-se, assim, que todos os pacientes que recebessem atendimento médico tivessem a mesma probabilidade de participar do estudo.

O instrumento de coleta de dados foi composto de um questionário estruturado pré-codificado (contendo informações da criança, de seu acompanhante e questões referentes à compreensão da prescrição) e de uma planilha para coleta de informações da prescrição (indicadores de prescrição e medicamentos prescritos).

As variáveis independentes do acompanhante foram: sexo, idade (em anos completos), grau de parentesco com a criança, cor da pele (classificada como branca ou não branca, com base na observação do entrevistador), escolaridade em anos de estudo e renda familiar (em reais divididos em quintis, onde o primeiro quintil referese aos $20 \%$ mais pobres, e o quinto aos $20 \%$ mais ricos). As variáveis independentes da criança foram sexo e idade (em anos completos).

As variáveis dependentes foram a prescrição de antimicrobianos (antimicrobianos mais prescritos e indicadores de prescrição); a compreensão da prescrição pediátrica quanto à indicação do fármaco, dose, intervalo de administração e duração do tratamento; a legibilidade da prescrição; e um indicador de assistência ao paciente. As prescrições de antimicrobianos de uso tópico foram consideradas no estudo somente para análise de compreensão da prescrição.

Os indicadores de prescrição e de assistência ao paciente preconizados pela Organização Mundial da Saúde (OMS) e avaliados no estudo foram extraídos do manual Como investigar el uso de medicamentos en los servicios de salud $(1993)^{8}$. Dentre os indicadores de prescrição, verificou-se a porcentagem de consultas em que é prescrito pelo menos um antimicrobiano de uso sistêmico, o número médio de medicamentos por prescrição de antimicrobianos e porcentagem de antimicrobianos prescritos pelo nome genérico. Dentre os indicadores de assistência ao paciente, foi verificado o tempo de consulta médica, o qual foi obtido através da utilização de cronômetro, sendo anotado em minutos o período que a criança permanecia em consulta.

Os medicamentos foram classificados segundo a Anatomical Therapeutical Chemical Classification System (ATC), preconizado pelo Drug Utilization Research Group (DURG) da OMS para estudo de utilização de medicamentos ${ }^{9}$.

Foi analisada a concordância da informação sobre o antimicrobiano prescrito, relatada pelo acompanhante que portava a prescrição médica, sendo as respostas classificadas em informação correta, incorreta ou ignorada.

Considerando que não existe um instrumento apropriado para medir a compreensão dos tratamentos indicados, criou-se um escore para avaliar a compreensão da prescrição, baseado nas variáveis conhecimento sobre a indicação, dose, intervalo e 
duração do tratamento prescrito. O valor do escore compreendeu de 4 (todas as respostas corretas) a 12 pontos (todas respostas incorretas ou ignoradas). Cada resposta correta acrescentou 1 (um) ponto ao escore e cada incorreta ou ignorada acrescentou 3 (três) pontos. Para composição do escore, as respostas incorretas e ignoradas foram consideradas como sendo equivalentes.

Uma vez que não existe recomendação validada para a avaliação deste desfecho, optou-se por dicotomizar o mesmo e classificar a compreensão como "adequada" ou "inadequada". A compreensão foi considerada como "adequada" quando o valor do escore foi menor do que 6,5; estando acima desse valor, era considerada "inadequada”. O ponto de corte de 6,5 pontos foi estabelecido por ser o equivalente à melhor pontuação (4 pontos) acrescida de 1 (um) desvio-padrão do escore $(2,5)$.

A prescrição foi considerada legível quando o acompanhante da criança estava apto a ler o nome do antimicrobiano, independente do grau de escolaridade. Acompanhantes que comunicaram não saber ler não foram considerados para a análise de legibilidade.

O tempo de consulta médica, baseado na média de tempo da consulta, foi categorizado em abaixo e acima de 5 minutos.

Os dados foram duplamente digitados no programa Epi Info 6.04. Após o processo de digitação foi feita a validação dos dados. As análises estatísticas do banco de dados foram feitas com o pacote estatístico Stata 9.2 (Stata Corp., College Station, TX: 2007).

O teste de tendência linear foi utilizado para avaliar associação das variáveis categóricas com os desfechos. O Teste $\mathrm{T}$ foi utilizado para análise das médias do escore de compreensão. Calculou-se por regressão de Poisson as razões de prevalência bruta e ajustada da compreensão adequada para as categorias das variáveis estudadas.

O presente trabalho foi aprovado pelo Comitê de Ética da Associação de Caridade Santa Casa do município de Rio Grande, $\mathrm{RS}$, local onde o projeto originalmente foi concebido. A Secretaria Municipal de Saúde e Meio Ambiente de Bagé-RS consentiu na realização do mesmo, após ser informada sobre os objetivos da pesquisa e declarada a ausência de conflitos de interesse. O propósito do estudo foi explicado aos acompanhantes das crianças e, somente após a concordância dos entrevistados e assinatura do termo de consentimento, foi dada continuidade à entrevista.

\section{Resultados}

Os atendimentos resultaram em 1.285 medicamentos prescritos, sendo 225 antimicrobianos e, destes, 209 para uso sistêmico. Do total de consultas incluídas no estudo, a proporção em que foi prescrito pelo menos um antimicrobiano sistêmico foi de $41,8 \%$. Nas prescrições contendo antimicrobianos, a média de medicamentos por receita foi de 2,9.

A Tabela 1 descreve a distribuição das consultas com prescrição de antimicrobianos, na população estudada, de acordo com características demográficas da criança. A prescrição de antimicrobianos difere por faixa etária $(p=0,003)$ e é maior com o aumento da idade. A média de idade das crianças assistidas foi de 4 anos ( $\mathrm{DP}=3,3$ ).

Do total de antimicrobianos, $81,8 \%$ foram prescritos pelo nome genérico. A Tabela 2 descreve o perfil de prescrição de antimicrobianos de acordo com a idade da criança. Os antimicrobianos mais frequentes para uso sistêmico foram amoxicilina $(53,9 \%)$ e a associação sulfametoxazol + trimetoprima $(19,4 \%)$, sendo a amoxicilina $\mathrm{o}$ antimicrobiano mais frequente em todas as faixas etárias.

As características socioeconômicas e demográficas do acompanhante da criança no local do estudo, bem como a prevalência de compreensão adequada para toda a amostra estão descritas na Tabela 3. Verifica-se uma tendência de a prescrição ser menos compreendida entre acompanhantes com menos de quatro anos de estudo ( $p=0,001)$, renda entre o primeiro e segundo quintil ( $p$ $=0,02)$ e não-brancos ( $p=0,03)$.

Avaliando-se apenas as consultas onde houve prescrição de antimicrobianos (n 
Tabela 1 - Distribuição das consultas com prescrição de antimicrobianos $(N=209)$ de acordo com as características demográficas das crianças nas unidades especializadas de saúde. Bagé, RS, 2007.

Table 1 - Distribution of appointments with antimicrobial prescription $(N=209)$ according to children's demographic characteristics in health clinics. Bage, RS - Brazil, 2007.

\begin{tabular}{lccc}
\hline Variável & $\begin{array}{c}\text { Consultas com } \\
\text { prescrição ATBs* } \\
N(\%)\end{array}$ & IC 95\% & Valor- $p$ \\
\hline Sexo & $110(41,8)$ & $35,8-47,8$ & $1,00 * *$ \\
$\quad$ Masculino $(n=263)$ & $99(41,8)$ & $35,4-48,1$ & \\
Feminino $(n=237)$ & & & $0,003 * * *$ \\
Idade & $62(36,3)$ & $29,0-43,5$ & \\
Até 1 ano $(n=171)$ & $74(41,8)$ & $34,4-49,1$ & \\
Entre 2 -5 anos $(n=178)$ & $73(48,3)$ & $40,3-56,4$ & \\
6 anos ou mais $(n=151)$ & & & \\
\hline
\end{tabular}

* Antimicrobianos / *Antimicrobial

** Qui-quadrado / ** Chi-square

*** Teste de tendência linear / *** Linear trend test

Tabela 2 - Distribuição do perfil de prescrição de antimicrobianos de acordo com a faixa etária nas unidades especializadas de saúde. Bagé, RS, 2007, ( $N=209)$.

Table 2 - Antimicrobial prescription profile according to age group in health clinics. Bage, RS - Brazil, 2007, $(N=209)$.

\begin{tabular}{lcccc}
\hline \multicolumn{5}{c}{ Faixa etária (em anos completos) } \\
\hline Antimicrobianos a $^{\text {a }}$ & $\begin{array}{c}\text { Até } 1 \text { ano } \\
\mathrm{N}(\%)\end{array}$ & $\begin{array}{c}2-5 \text { anos } \\
\mathrm{N}(\%)\end{array}$ & $\begin{array}{c}6 \text { anos }+ \\
\mathrm{N}(\%)\end{array}$ & Total \\
\hline Amoxicilina & $25(40,3)$ & $42(56,7)$ & $44(60,3)$ & 111 \\
Benzilpenicilina & $7(11,3)$ & $8(10,8)$ & $5(6,8)$ & 20 \\
Benzatina & & & & \\
Cefalexina & $2(3,2)$ & $4(5,4)$ & $3(4,1)$ & 9 \\
Smx/Tmp & $14(22,6)$ & $14(18,9)$ & $12(16,4)$ & 40 \\
Outros ATBs & $14(22,6)$ & $6(8,1)$ & $9(12,3)$ & 29 \\
Total & $62(100)$ & $74(100)$ & $73(100)$ & 209 \\
\hline
\end{tabular}

${ }^{a}$ Classificados pela ATC - Anatomical Therapeutic Chemical Classification System

b Sulfametoxazol + trimetoprima

c ATBs = antimicrobianos

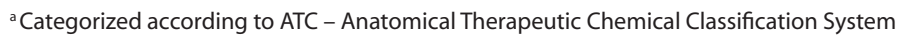

${ }^{b}$ Trimethoprim/Sulfamethoxazole association

${ }^{c}$ ATBs $=$ antimicrobials

= 209) com as variáveis estudadas (Tabela 4), verifica-se, em análise bruta, que a compreensão adequada esteve associada aos acompanhantes com melhor renda ( $p$ $=0,03)$, brancos $(p=0,007)$ e com nível de escolaridade e tempo de consulta maior, estando estas associações com significância estatística limítrofe $(p=0,05)$. Após ajuste entre si das variáveis analisadas, a compre- ensão adequada permaneceu associada apenas à cor da pele $(p=0,02)$ e tempo de consulta $(p=0,03)$. Esta diferença na análise ajustada, provavelmente, foi atribuída à diminuição do tamanho da amostra, quando consideramos somente as prescrições com antimicrobianos.

A média do tempo de consulta entre os pediatras do serviço foi de 5 minutos e 30 
Tabela 3 - Descrição das características sociodemográficas dos acompanhantes das crianças nas unidades especializadas em saúde e prevalência de compreensão adequada das prescrições pediátricas ( $N=500)$. Bagé, RS, 2007.

Table 3 - Socio-demographic characteristics of the person accompanying the child at health clinic and prevalence of appropriate understanding of pediatric prescription ( $\mathrm{N}=500)$. Bage, RS - Brazil, 2007.

\begin{tabular}{|c|c|c|c|c|}
\hline Variável & $\mathrm{N}$ & $\%$ & $\begin{array}{l}\text { Compreensão } \\
\text { adequada (\%) }\end{array}$ & $P$ \\
\hline Sexo & & & & $0,22^{\mathrm{a}}$ \\
\hline Mulheres & 433 & 86,6 & 53,7 & \\
\hline Homens & 67 & 13,4 & 61,7 & \\
\hline Idade (anos) & & & & $0,53^{b}$ \\
\hline $15-19$ & 35 & 7,0 & 45,7 & \\
\hline $20-29$ & 180 & 36,0 & 63,3 & \\
\hline $30-39$ & 185 & 37,0 & 60,5 & \\
\hline 40 - ou mais & 100 & 20,0 & 61,0 & \\
\hline Cor da pele & & & & $0,03^{\mathrm{a}}$ \\
\hline Branca & 341 & 68,0 & 63,9 & \\
\hline Não branca & 159 & 31,8 & 53,5 & \\
\hline Grau de Parentesco & & & & $0,02^{b}$ \\
\hline Mãe & 432 & 86,4 & 62,5 & \\
\hline Pai & 31 & 6,2 & 48,4 & \\
\hline Avó/ avô & 23 & 4,6 & 52,2 & \\
\hline Tia / tio & 6 & 1,2 & 83,3 & \\
\hline Outro & 8 & 1,6 & 12,5 & \\
\hline Escolaridade (anos de estudo) & & & & $0,001^{\prime}$ \\
\hline $0-4$ & 73 & 14,6 & 47,9 & \\
\hline $5-8$ & 205 & 41,0 & 57,6 & \\
\hline 9 - ou mais & 222 & 44,4 & 67,6 & \\
\hline Quintil de renda familiar (R\$) & & & & $0,02^{b}$ \\
\hline Primeiro (até 280,00 ) & 102 & 20,4 & 55,9 & \\
\hline Segundo $(281,00-370,00)$ & 133 & 26,6 & 56,4 & \\
\hline Terceiro $(371,00-480,00)$ & 98 & 19,6 & 61,2 & \\
\hline Quarto $(481,00-670,00)$ & 75 & 15,0 & 60,0 & \\
\hline Quinto $(671,00-4000,00)$ & 92 & 18,4 & 71,7 & \\
\hline
\end{tabular}

a: Qui-quadrado / a: Chi-square

$\mathrm{b}$ : teste de tendência linear / $b$ : Linear trend test

segundos (DP = 2'30"; amplitude de 1-19). As consultas com tempo superior a $5 \mathrm{mi}-$ nutos obtiveram melhor compreensão da prescrição pediátrica pelo acompanhante da criança, tanto para o total de consultas assistidas ( $p=0,004)$, quanto para aquelas com pelo menos um antimicrobiano prescrito $(p=0,03)$. A média de tempo de consulta para os níveis de escolaridade entre zero - 4 anos; 5-8 anos e 9 anos ou mais de estudo, foi de 5'13"; 5'14" e 5'51", respectivamente, havendo uma tendência para o aumento do tempo de consulta à medida que aumentava a escolaridade $(p=0,02)$.
Foram consideradas de compreensão adequada 58,9\% das prescrições contendo ao menos um antimicrobiano, ou seja, com valor do escore menor que 6,5 pontos.

Das consultas onde foi prescrito antimicrobiano, 97,8 \% das prescrições continham a dose, $93,3 \%$ o intervalo de administração do medicamento e 87,5\% a duração do tratamento especificada. Quanto à legibilidade, 30,1\% dos acompanhantes não conseguiram ler o nome do antimicrobiano, sendo a prescrição classificada como ilegível. Apenas 2,4\% dos entrevistados mencionaram ser anal- 
Tabela 4 - Descrição da compreensão adequada de acordo com as variáveis estudadas e Razões de Prevalência (RP) bruta e ajustada obtidas por Regressão de Poisson. ( $N$ = 209). Bagé, RS, 2007.

Table 4 - Appropriate understanding of prescription according to variables assessed and crude and adjusted prevalence ratios obtained by Poisson regression. ( $\mathrm{N}=209)$ Bage, RS - Brazil, 2007.

\begin{tabular}{|c|c|c|c|c|c|c|}
\hline \multirow[t]{2}{*}{ Variável } & \multirow[t]{2}{*}{$\%$} & \multirow{2}{*}{$\begin{array}{l}\text { \% Compreensão } \\
\text { adequada }\end{array}$} & \multirow{2}{*}{$\begin{array}{l}\text { Análise Bruta } \\
\text { RP (IC 95\%) }\end{array}$} & \multicolumn{3}{|c|}{ Análise ajustada } \\
\hline & & & & $\mathrm{p}^{*}$ & RP (IC 95\%) & $\mathrm{p}^{*}$ \\
\hline Escolaridade & & & & 0,05 & & 0,33 \\
\hline $0-4$ anos & 36,4 & 50,0 & 1,00 & & 1,00 & \\
\hline $5-8$ anos & 37,8 & 55,0 & $0,90(0,59-1,37)$ & & $0,96(0,62-1,47)$ & \\
\hline 9 anos + & 25,8 & 67,1 & $0,66(0,41-1,06)$ & & $0,79(0,47-1,33)$ & \\
\hline Renda (quintis) & & & & 0,03 & & 0,18 \\
\hline 1 & 22 & 52,2 & 1,00 & & 1,00 & \\
\hline 2 & 27,3 & 52,6 & $0,99(0,66-1,49)$ & & $1,10(0,74-1,64)$ & \\
\hline 3 & 19,6 & 58,5 & $0,87(0,54-1,39)$ & & $0,94(0,58-1,55)$ & \\
\hline 4 & 13,4 & 64,3 & $0,75(0,42-1,34)$ & & $0,84(0,46-1,54)$ & \\
\hline 5 & 17,7 & 73,0 & $0,56(0,31-1,04)$ & & $0,71(0,37-1,34)$ & \\
\hline Cor da pele & & & & 0,007 & & 0,02 \\
\hline Branca & 66,0 & 65,2 & 1,00 & & 1,00 & \\
\hline Não-branca & 34,0 & 46,5 & $1,53(1,12-2,11)$ & & $1,44(1,05-2,00)$ & \\
\hline Tempo de consulta & & & & 0,05 & & 0,03 \\
\hline$<5$ minutos & 41,2 & 53,2 & 1,00 & & 1,00 & \\
\hline$>5$ minutos & 58,9 & 67,1 & $0,70(0,49-1,00)$ & & $0,68(0,48-0,97)$ & \\
\hline
\end{tabular}

* Teste de Wald / * Wald's test

fabetos e por isso não leram o nome do medicamento.

Em relação à concordância das informações sobre antimicrobianos relatadas pelo acompanhante da criança portadora da prescrição (Tabela 5), quase metade das pessoas informou incorretamente ou não soube informar a dose, e o mesmo se repetiu para um terço quanto à variável intervalo de administração. Aproximadamente 30\% informaram incorretamente ou não souberam informar a duração do tratamento.

\section{Discussão}

Falhas no seguimento da terapia farmacológica estão entre os principais fatores que induzem a um processo de resistência antimicrobiana aos fármacos, o que compromete a saúde da população e leva a onerosos gastos para o poder público ${ }^{5,10}$.

O percentual de consultas, no qual foi prescrito pelo menos um antimicrobiano $(41,8 \%)$ demonstra que o indicador de racionalidade está fora dos parâmetros

Tabela 5 - Classificação das informações sobre antimicrobianos relatadas pelos acompanhantes das crianças $(\mathrm{N}=209)$. Bagé, RS, 2007.

Table 5 - Antimicrobial information as reported by the person accompanying the child ( $N=209)$. Bage, RS - Brazil, 2007.

Relato das informações do antimicrobiano prescrito

\begin{tabular}{lcccccccc}
\hline & \multicolumn{2}{c}{ Indicação } & \multicolumn{2}{c}{ Dose } & \multicolumn{3}{c}{ Intervalo de administração } & \multicolumn{2}{c}{ Duração do tratamento } \\
& $\mathrm{N}$ & $\%$ & $\mathrm{~N}$ & $\%$ & $\mathrm{~N}$ & $\%$ & $\mathrm{~N}$ & $\%$ \\
\hline Correta & 166 & 79,4 & 119 & 57 & 134 & 64,1 & 148 & 70,8 \\
Incorreta & 12 & 5,8 & 26 & 12,4 & 26 & 12,4 & 21 & 10,1 \\
Não sabem & 31 & 14,8 & 64 & 30,6 & 49 & 23,5 & 40 & 19,1 \\
Total & 209 & 100 & 209 & 100 & 209 & 100 & 209 & 100 \\
\hline
\end{tabular}


preconizados pela OMS $(20-30 \%)^{8}$. Outros trabalhos no Brasil, realizados em unidades básicas de saúde na população infantil, encontraram valores entre $29-44,6 \%^{11,12}$. Existem evidências de que no contexto da utilização de antimicrobianos, a especialidade médica que mais prescreve este grupo farmacológico é a pediatria ${ }^{13,14}$.

Alguns pesquisadores têm mencionado que a idade da mãe e o seu nível de escolaridade, também são fatores condicionantes para a maior prescrição de antimicrobianos aos seus filhos ${ }^{15,16}$.

As consultas com prescrições de antimicrobianos sistêmicos apresentaram uma média elevada no número de medicamentos prescritos $(2,9)$, o que pode ser considerado um risco potencial para interações medicamentosas ${ }^{12} \mathrm{e}$ incompreensão da prescrição ${ }^{7}$. Um estudo realizado em Araraquara (SP), em crianças com idade entre um a cinco anos, apresentou valor aproximado $(2,7)^{11}$. De acordo com a OMS, a média aceitável de medicamentos por prescrição médica estaria entre $1,3-2,2^{8}$.

Verificou-se um elevado percentual de prescrição de antimicrobianos pelo nome genérico $(81,8 \%)$, comparado a outros estudos realizados em serviço de saúde com atendimento pediátrico $(32 \%-45,2 \%)^{11,12}$. No entanto, este indicador encontra-se em desacordo com a legislação, a qual preconiza no âmbito do Sistema Único de Saúde que $100 \%$ dos medicamentos sejam prescritos pela Denominação Comum Brasileira ou, na sua falta, pela Denominação Comum Internacional ${ }^{17}$. A padronização nos hábitos de prescrição pela denominação genérica pode proporcionar ao acompanhante da criança uma melhor compreensão do nome do medicamento e indicação clínica do mesmo.

Neste estudo foram prescritos 13 antimicrobianos distintos. O grupo farmacológico penicilinas representou mais da metade das prescrições. Os antimicrobianos mais prescritos foram a amoxicilina, seguida da associação de sulfametoxazol-trimetoprima, ambos de amplo espectro de ação. Vários trabalhos têm demonstrado a vasta utiliza- ção desses dois antimicrobianos no âmbito comunitário, cujas variações na frequência de utilização compreendem valores entre 35,8 a $54 \%$ e 12,1 a $26,8 \%$, respectivamente ${ }^{11,14,15,18}$. Os antimicrobianos prescritos não padronizados pela Lista Municipal de Padronização foram cefadroxila, a associação amoxicilina + ácido clavulônico, cloranfenicol e azitromicina, também considerados de amplo espectro. A utilização excessiva de antimicrobianos de amplo espectro aumenta os riscos de pressão seletiva a vários patógenos $^{15,19}$.

Houve uma tendência de os antimicrobianos serem mais prescritos com o aumento da idade, mostrando-se esta relação inversa à encontrada em outros estudos, onde foi observada diminuição da prescrição em crianças com uma faixa etária maior ${ }^{15,18}$. Pesquisas apontam que a utilização de antimicrobianos em crianças é duas vezes maior comparada à utilização em adultos, e que a faixa etária inferior a cinco anos recebe significativa prescrição de antimicrobianos ${ }^{18,20}$. Algumas hipóteses podem estar de acordo com a tendência de os antimicrobianos serem mais prescritos à medida que aumenta a idade: desmame a partir do primeiro ano de vida, levando a uma diminuição das imunoglobulinas, favorecendo, consequentemente, o aparecimento de infecções e o maior contato com o mundo exterior à medida que a criança cresce, proporcionando o contato com agentes infecciosos, principalmente em creches ou pré-escola ${ }^{21}$.

A maioria dos acompanhantes das crianças eram mães jovens, com escolaridade suficiente para a habilidade de leitura. No entanto, há de se considerar que, no Brasil, $59 \%$ dos alunos com até quatro anos de estudo não apresentam habilidades de leitura compatíveis com o nível de letramento e são enquadrados como analfabetos funcionais, uma vez que não usam a linguagem escrita como elemento essencial de sua vida ${ }^{22}$.

A quase totalidade das informações sobre dose $(97,85 \%)$, intervalo de administração (93,3\%) e duração do tratamento $(87,5 \%)$, esteve presente no conteúdo das 
prescrições de antimicrobianos. Abrantes e col. (2007) observaram, em estudo em unidade básica de saúde, resultados similares com valores de 99,6\%, 98,9\% e 89,3\%, respectivamente ${ }^{14}$.

Informações omitidas do conteúdo das prescrições podem se constituir num problema, especialmente para pessoas com pouca escolaridade, ou quando um esquema de múltiplas drogas foi prescrito, além de representar um dos tipos mais comuns de erro de medicação, contribuir para a não adesão ao tratamento ${ }^{5}$ e dificultar a dispensação de medicamentos no serviço de saúde ${ }^{14}$. A prescrição é um documento fornecido por profissional habilitado e determina as condições em que o medicamento deve ser utilizado ${ }^{23}$. A qualidade da prescrição médica é um dos fatores que determinam como os usuários de saúde, neste caso os acompanhantes das crianças, utilizam os medicamentos ${ }^{5}$.

O reflexo da omissão de informações na consulta médica pode ter influenciado a concordância das informações relatadas pelo acompanhante da criança com a prescrição de antimicrobiano. Cerca da metade das pessoas informaram incorretamente ou não souberam informar a dose (43\%); o mesmo se repetiu para um terço quanto à variável intervalo de administração (36\%), e cerca de $30 \%$ não informaram corretamente a duração do tratamento. $\mathrm{O}$ não entendimento dos dados técnicos da prescrição torna-se preocupante, uma vez que o indicador de prescrição de antimicrobianos aponta valores superiores ao preconizado pela OMS, o que pode levar a erros de medicação e consistir em agravo para o fenômeno de resistência aos antimicrobianos. Além disso, os dados sugerem que a comunicação pediatra-acompanhante tenha sido limitada, enfatizando a necessidade de desenvolver, no serviço de saúde, metodologias educativas que assegurem a transmissão e captação das informações. Esta hipótese é confirmada por outros pesquisadores, os quais mencionam que um dos fatores da incompreensão da prescrição é a relação paciente-prescritor, e que os pacientes devem ser encorajados a sanar suas dúvidas em consulta médica ${ }^{4,24,25}$. Uma compreensão adequada da prescrição favorece o sucesso do tratamento e pode evitar o fenômeno de resistência microbiana.

Ao ser associada a compreensão da prescrição pediátrica com o grau de escolaridade e renda do acompanhante, tanto no total de consultas quanto somente naquelas com prescrição de antimicrobianos, verificaram-se maiores prevalências de compreensão adequada para quem tinha mais escolaridade e maior renda. No entanto, em análise ajustada, o poder dessa associação diminuiu nas prescrições contendo antimicrobianos, provavelmente pelo menor tamanho da amostra, não sendo essa diferença estatisticamente significativa.

Pesquisas nacionais ${ }^{6-26}$ e internacionais ${ }^{3,7}$ que relacionaram a compreensão da informação escrita indicam a escolaridade como fator importante para a boa compreensão. Estes trabalhos, ao verificarem a compreensão dos usuários de unidades de saúde quanto às informações contidas em rótulos de medicamentos ou em prescrições médicas após consulta, apontaram uma prevalência de incompreensão da informação escrita entre 29 - 63\% ${ }^{3,6,7,26}$.

Neste estudo, a compreensão inadequada correspondeu a $41,1 \%$. Há de se considerar que, embora o indivíduo declare corretamente as instruções de uso do medicamento, às vezes não as compreende, demonstrando uma deficiência cognitiva. Um estudo que analisou a compreensão de bulas de medicamentos demonstrou que $70,7 \%$ dos usuários do serviço, com baixa escolaridade, declararam adequadamente as instruções da bula. Por outro lado, somente $34,7 \%$ demonstraram o número correto de comprimidos a ser tomado diariamente ${ }^{7}$.

Existe um consenso, formado a partir de diversas evidências, de que o nível de escolaridade está positivamente correlacionado com a renda. No Brasil, a taxa de analfabetismo nos domicílios cujo rendimento é superior a dez salários mínimos é de apenas $1,4 \%$ e, naqueles cujo rendimento é inferior a um salário mínimo, é de quase $29 \%$, de- 
monstrando que a renda influencia no acesso à educação ${ }^{22}$. Da mesma forma, dados demonstram que indivíduos não-brancos possuem menos recursos financeiros e, em consequência, menores oportunidades de estudo $^{27}$. Assim, percebe-se a relação com a maior dificuldade de compreensão das informações cedidas pelo serviço de saúde.

O tempo de consulta médica esteve abaixo do preconizado (15 minutos) ${ }^{8}$. Este estudo pôde demonstrar que esta variável é um fator determinante na compreensão da prescrição pediátrica, pois mesmo estando a média de tempo distante do preconizado, a maior prevalência de compreensão adequada foi a média de tempo acima de 5 minutos. O tempo de consulta foi diretamente proporcional à escolaridade, sendo as maiores médias de tempo de consulta para os acompanhantes com maior escolaridade. No entanto, deveria ser o inverso, já que mães jovens, com menos experiência e menor nível de escolaridade necessitam de mais tempo de consulta e suporte do pediatra ${ }^{16}$. Seriam necessários estudos qualitativos para a avaliação dos fatores que influenciam o tempo de consulta.

Nos locais em estudo, todas as prescrições foram manuscritas, fator este que aumenta os problemas quanto à legibilidade e contribui para a incompreensão da prescrição. Sistemas eletrônicos de prescrição pediátrica têm sido desenvolvidos no intuito de reduzir os erros de prescrição e melhorar a legibilidade pelo acompanhante da criança ${ }^{28}$. A legibilidade influencia a comunicação e pode interromper ou alterar o processo de assistência ao paciente ${ }^{23}$. Falhas na leitura e incompreensão da informação escrita podem contribuir para erros de medicação; logo, é necessário que esta informação seja fornecida com letra maior, de forma organizada, lógica e apropriada à habilidade de leitura, de forma a facilitar a compreensão do tratamento ${ }^{4}$.

Uma limitação do estudo foi o fato de o mesmo ter sido realizado em apenas três Unidades de Saúde, em virtude de somente estas disporem de serviço especializado em pediatria, contando com o profissional pe- diatra. Além disso, embora se tenha estimado uma amostra de tamanho suficiente para as associações entre as variáveis com seus desfechos, considerou-se uma prevalência de utilização de antimicrobianos insuficiente para as análises ajustadas, mascarando os fatores determinantes da compreensão da prescrição de antimicrobianos. Sugere-se a reprodução desse trabalho em todas as Unidades de Saúde Pública do Município para que haja uma representatividade da compreensão das prescrições pediátricas de antimicrobianos e, em consequência, um maior tamanho de amostra.

\section{Considerações finais}

Neste estudo, verificou-se que a escolaridade, renda e cor da pele do acompanhante da criança, bem como o tempo de consulta, estiveram diretamente associados à compreensão da prescrição de medicamentos e que, quanto maior a escolaridade e renda do acompanhante da criança e o tempo de consulta, melhor a compreensão, sendo esta maior para indivíduos brancos.

A compreensão da prescrição de antimicrobianos poderia ter sido melhor se a totalidade dos medicamentos tivesse sido prescrita pela denominação genérica, facilitando a familiarização com o tratamento, nome do medicamento e a indicação do mesmo; também se a caligrafia estivesse adequada à total legibilidade e se o índice de politerapia estivesse reduzido nas prescrições contendo antimicrobianos.

Falhas na terapia farmacológica podem ser evitadas com a correta compreensão da terapêutica antimicrobiana por parte do acompanhante da criança, já que fatores relacionados ao prescritor, como a clareza das informações verbais e escritas, fornecidas durante a consulta médica, e os referentes ao acompanhante da criança, tal como a dificuldade em questionar durante a consulta, podem interferir na compreensão da prescrição. Em função disso, os profissionais de saúde devem estar preparados para promover medidas de educação em saúde, tais como o fornecimento de orientações 
verbal e escrita quanto ao correto uso dos antimicrobianos no momento da consulta e dispensação de medicamentos. Assim, pode-se assegurar a correta compreensão das informações destinadas aos usuários do serviço, independentemente do grau de escolaridade.

A padronização da informação escrita em Unidades de Saúde pode também ser uma estratégia que aumente a consistência do conteúdo da informação e melhore a compreensão da prescrição pediátrica, evitando-se erros de medicação e podendose, assim, não só contribuir para uma melhor adesão ao tratamento, mas minimizar o fenômeno de resistência antimicrobiana.

\section{Referências}

1. Ministry of Health (MOH). Clinical Practice Guidelines Use antibiotics in pediatric care. Singapore: Ministry of Health; 2002.

2. Marin N, Luiza VL, Osório de Castro CGS, Machado dos Santos S (org.). Assistência Farmacêutica para gerentes municipais. Rio de Janeiro: OPAS/OMS; 2003.

3. Wolf MS, Davis TC, ShrankW, Rapp DN, Bass PF, Connor UM et al. To err is human: patient misinterpretations of prescription drug label instructions. Patient Educ Couns 2007; 67(3): 293-300.

4. Shrank W, Avorn J, Rolon C, Shekelle P. Effect of Content and Format of Prescription Drug Labels on Readability, Understanding, and Medication Use: A Systematic Review. Ann of Pharmacotherapy 2007; 41(5): 783-801.

5. World Health Organization (WHO). How to investigate the use of medicines by consumers. Geneva: WHO 2004. Disponível em http:/ / www.who.int/medicines/en/ [Assessado em 25 de novembro de 2007].

6. Sano PY, Masotti RR, dos Santos AAC, Cordeiro JA. Avaliação do nível de compreensão da prescrição pediátrica. J Pediatr 2002; 78(2): 140-45.

7. Davis TC, Wolf MS, Bass PF, Thompson JA, Tilson HH, Neuberger M, et al. Literacy and Misunderstanding Prescription Drug Labels. Ann. Intern Med 2006; 145(12): 887-94.

8. Organización Mundial de la Salud (OMS). Como investigar el uso de medicamentos en los servicios de salud. Ginebra: OMS/DAP; 1993.

9. World Health Organization. Collaborating Center for Drug Statistics Methodology. Anatomical Therapeutic Chemical (ATC) classification index. Oslo: World Health Organization; 2000.

10. Pichichero, ME. Understanding antibiotic overuse for respiratory tract infections in children. Pediatrics 1999; 104(6): 1384-8.

11. Fegadolli C, Simões MJS, Jorge IC, Tanaka KSS. Antibioticoterapia em crianças na prática diária em Unidades de Saúde de Araraquara - SP, 1997. Rev Ciênc Farm 1999; 20(1): 124-42.
12. Fegadolli C, Mendes IJM, Simões MJS. Avaliação da prescrição médica em pediatria, baseada nos indicadores do uso de medicamentos selecionados pela OMS em município do interior do Estado de São Paulo. Rev Cienc Farm 2002; 23(2): 239-54.

13. Santos V, Nitrini SMOO. Indicadores do uso de medicamentos prescritos e de assistência ao paciente de serviço de saúde. Rev Saúde Pública 2004; 38(6): 819-26.

14. Abrantes PM, Magalhães SMS, Acúrcio FA, Sakurai E. Avaliação da qualidade das prescrições de antimicrobianos dispensadas em unidades públicas de saúde de Belo Horizonte, Minas Gerais, Brasil, 2002. Cad Saúde Pública 2007; 23(1): 95-104.

15. Zhang L, Mendoza R, Costa MMG, Ottoni EJG, Bertaco AS, Santos JCH et al. Antibiotic use in Community-Based Pediatric Outpatients in Southern Region of Brazil. $J$ Trop Pediatr 2005; 51(5): 304-9.

16. Zaffani S, Cussolin L, Meneghelli G, Gangemi M, Murgia V, Chiamenti G et al. An analysis of the factors influencing the pediatrician-parent relationship: the importance of the socio-demographic characteristics of the mothers. Child Care Health Dev 2005; 31(5): 575-80.

17. Decreto 3181. Regulamenta a lei $n^{\circ} 9.797 / 1999$ que dispõe sobre a vigilância sanitária, estabelece o medicamento genérico, dispõe sobre a utilização de nomes genéricos em produtos farmacêuticos e dá outras providências. Diário Oficial da União, 24 de setembro de 1999.

18. Berquó LS, Barros AJD, Lima RC, Bertoldi AD. Utilização de antimicrobianos em uma população urbana. Rev Saúde Pública 2004; 38(2): 239-46.

19. Wannmacher L. Uso indiscriminado de antibióticos e resistência microbiana: uma guerra perdida? Uso Racional de Medicamentos: Temas Selecionados. Brasília: OPAS/OMS; 2004: 1(4).

20. Wang EEL, Einarson TR, Kellner JF, Conly JM. Antibiotic Prescribing for Canadian Preschool Children: evidence of Overprescribing for Viral Respiratory Infections. Clin Infect Dis 1999; 29: 155-60. 
21. Sarinho ESC, Aroucha M, Melo SA, Xavier TL. Uso abusivo de antimicrobianos em pediatria: estudo de crianças de 0 à 2 anos atendidas no ambulatório de puericultura do hospital das clínicas da UFPE. Rev Bras Saúde Mater Infant 1993; 7(1): 25-28.

22. Instituto Nacional de Estudos e Pesquisas Educacionais Anísio Teixeira (INEP). Manual do Analfabetismo no Brasil. Ministério da Educação; 2001.

23. Aguiar G, Júnior LAS, Ferreira MMA. Ilegibilidade e ausência de informações nas prescrições médicas: fatores de risco relacionados a erros de medicação. Rev Bras Promoção a Saúde 2006; 19(2): 84-91.

24. Britten N, Stevenson FA, Barry CA, Barber N, Bradley CP. Misunderstandings in prescribing decisions in general practice: qualitative study. Br Med J 2000; 320: 484-8.

25. DiMatteo MR. The role of effective communication with children and their families in fostering adherence to pediatric regimens. Patient Educ Couns 2004; 55: 339-44.
26. Silva T, Schenkel EP, Mengue SS. Nível de informação a respeito de medicamentos prescritos a pacientes ambulatoriais de hospital universitário. Cad Saúde Pública 2000; 16(2): 449-55.

27. Lopes, F . Para além da barreira dos números: desigualdades raciais e saúde. Cad Saúde Pública 2005; 21(5): 1595-1601.

28. Official Journal of the American Academy of Pediatrics. Electronic Prescribing Systems in Pediatrics: The Rationale and Functionality Requirements. Council on Clinical Information Technology. Pediatrics 2007; 199(6): 1229-31.

Recebido em: 06/01/09 Versão final reapresentada em: 15/06/09 Aprovado em: 23/06/09 